\title{
Adding Pieces to the CO/Pt(111) Puzzle: The Role of Dispersion
}

\author{
Patanachai Janthon $^{a, b, c, d}$, Francesc Viñes ${ }^{a}$, Jakkapan Sirijaraensre ${ }^{b, c}$, \\ Jumras Limtrakul ${ }^{e}$, Francesc Illas, ${ }^{a, *}$ \\ ${ }^{a}$ Departament de Ciència dels Materials i Química Física \& Institut de Química Teòrica i Computacional \\ (IQTCUB), Universitat de Barcelona, C/ Martí i Franquès 1, 08028 Barcelona, Spain. \\ ${ }^{b}$ Department of Chemistry, Faculty of Science, Kasetsart University, Bangkok 10900, Thailand. \\ ${ }^{c}$ Center for Advanced Studies in Nanotechnology for Chemical, Food and Agricultural Industries, KU Institute \\ for Advanced Studies, Kasetsart University, Bangkok 10900, Thailand. \\ ${ }^{d}$ Faculty of Science, Chandrakasem Rajabhat University, Bangkok, 10900, Thailand. \\ ${ }^{e}$ Department of Materials Science and Engineering, School of Molecular Science and Engineering, \\ Vidyasirimedhi Institute of Science and Technology, Rayong 21210, Thailand.
}

\begin{abstract}
The so called CO/Pt(111) puzzle, the experimentally proven preference of $\mathrm{CO}$ to adsorb on top site on $\mathrm{Pt}(111)$ surface versus the standard density functional theory (DFT) methods prediction for three-fold hollow sites, was alleged to be solved by properly leveling $\mathrm{CO}$ frontier molecular orbitals. However, the subtle energy difference between top and hollow sites is of the same order of the possible contribution of dispersive forces on this interaction. Here, the role of dispersion on this system is investigated by considering the PBE, PBEsol, RevPBE, RPBE, and SOGGA11 generalized gradient approximation (GGA) based exchange correlation functionals, non-separable functionals such as N12, and the TPSS and M06-L meta-GGA type functionals together to D2, D3, D3BJ and MBD dispersion corrections. Results reinforce the advice of using M06-L for a correct description of CO adsorption site preference even if including dispersion leads to a change of site and a noticeable overestimation of the adsorption energy indicating the presence of error compensations effects. The present results also highlight that dispersion contributes in bridging the preference gap between top and hollow sites when other functionals are used. Dispersive forces play a role in site preference for $\mathrm{CO}$ on $\mathrm{Pt}(111)$ and it is likely that a similar situation is encountered on other late transition metals. Therefore, dispersion is to be considered to reach a complete unbiased description of $\mathrm{CO}$ adsorption on metals. Nevertheless, including dispersion leads to adsorption energy values which overestimate the experimental value indicating limitations of the existing, widely used, density functionals.

*Corresponding Author: francesc.illas@ub.edu
\end{abstract}




\section{Introduction}

Late transition metals are key components in several industrial chemical processes with unique electronic properties exploited in homogenous ${ }^{1}$ and heterogeneous catalysis. ${ }^{2,3}$ To highlight one among many, a challenging application is the usage of late transition metal based catalysts for the production of renewable fuels, hydrogen being one of the outstanding energy carriers. Nowadays $\mathrm{H}_{2}$ production can run on green chemistry, but scarce and so expensive catalytic materials, such as platinum, are used as an important active phase in hydrogen generation fuel cells. ${ }^{4}$ However, as it is also the case of many transition metal based catalysts, $\mathrm{Pt}$ is easily poisoned by carbon monoxide $(\mathrm{CO})$; it adsorbs intact on the $\mathrm{Pt}$ surface ${ }^{5}$ forming a strong chemical bond with the surface. ${ }^{6}$

Earlier measurements from temperature programmed desorption (TPD) experiments reported an estimate adsorption energy of $1.43 \mathrm{eV}^{7}$ not so far from the most recent estimates from velocity selected residence time methodology yielding $1.47 \pm 0.04 \mathrm{eV}$ at a very low coverage regime of $<0.03$ monolayers $(\mathrm{ML}){ }^{8}$ Therefore, the nature and strength of $\mathrm{CO}$ chemisorption on $\operatorname{Pt}(111)$ is well established and out of debate. This is also the for the adsorption site with a clear preference for CO on top of Pt surface atoms. ${ }^{9-11}$ These well defined properties make $\mathrm{CO}$ on $\mathrm{Pt}(111)$ especially suited to benchmark theoretical approaches and surface models. Surprisingly, density functional theory (DFT) based calculations on periodic models using different flavors - from the local density approximation (LDA) to various generalized gradient approximation (GGA) approaches - of standard exchange correlation functionals fail to predict the experimentally observed site favoring instead the three-fold $f c c$ hollow site. The same result whether all electrons are considered or employing pseudopotentials to describe the effect of core electrons on the valence electron density. The resulting situation was reviewed several years ago and lead to what is now generally known as "The CO/Pt(111) Puzzle"."

This puzzle, on an apparently simple system, triggered subsequent studies aimed at providing a proper explanation for the disagreement, a suitable modification of existing methods, a test for new methods and, eventually, a solution of the problem. ${ }^{13,14}$ To date it is clear that the failure arises from a wrong leveling of CO highest occupied molecular orbital (HOMO) and lowest unoccupied molecular orbital (LUMO) with respect Pt band structure, ${ }^{15,16}$ the $\mathrm{CO}$ bonding to transition metal surfaces can be described via a $\sigma$-donation from $\mathrm{CO} 5 \sigma \mathrm{HOMO}$ to the metal surface, and backbonding from the metal to the unoccupied 
CO $2 \pi^{*}$ LUMO as put forward by Blyholder more than 50 years ago. ${ }^{17}$ The relative strength of these two synergetic interactions depends on the local topology of the adsorption site and the electronic structure of the metal. The inherent self-interaction error of all semilocal exchange-correlation (xc) potentials makes that the partially occupied $2 \pi^{*}$ orbital lies too low in energy, thus artificially enhancing the backbonding contribution which in turn is strongest on hollow sites, thus rationalizing the unphysical preference for $\mathrm{CO}$ adsorption at face centered cubic $(f c c)$ hollow sites of $\mathrm{Pt}(111) .{ }^{18}$ Indeed, the over-contribution of backbonding does also explain the generally too large adsorption energies with these semilocal xc potentials which can be overestimated by more than $0.4 \mathrm{eV} .{ }^{18-20}$ Hybrid xc functionals such as PBE0, ${ }^{21} \mathrm{~B} 3 \mathrm{LYP},{ }^{22}$ and $\mathrm{HSE}^{23}$ exhibit a reduced self-interaction error and lead to a more accurate description of thermochemistry, and so are expected to counteract the exceedingly large adsorption energy and incorrect molecular orbital leveling description of GGA type functionals. With this type of functionals the problem seems be fixed, at least for $\mathrm{CO}$ on $\mathrm{Cu}(111)$ and $\mathrm{Rh}(111)$, but remains open for $\mathrm{CO}$ on $\mathrm{Pt}(111) .{ }^{19}$ The PBE0 calculations of Wang et al. ${ }^{24}$ for a $\mathrm{CO}$ on $\operatorname{Pt}(111)$ using a $(\sqrt{3} \times \sqrt{3})-\mathrm{R} 30^{\circ}$ supercell correctly predict the preference for the top site yet using the PBE optimized geometry. In fact, the systematic study of Stroppa and $\mathrm{Kresse}^{25}$ using GGA and hybrid functionals and covering various surfaces find preference for the top site using B3LYP, but $f c c$ using HSE. Other authors highlighted the use of all electron relativistic calculations to cure the leveling problem. ${ }^{26}$ Interestingly, the M06-L meta-GGA functional appears to predict the correct adsorption site and also reproduces rather well the experimental adsorption energy. ${ }^{27}$ The authors stated that inclusion of the kinetic energy density in M06-L ansatz allowed for a better description of single orbital states and of electron density decays, being both important for orbital levelling and surface modelling, where electron density exponentially decays to zero. This as well allows for a more realistic treatment of large exchange interactions, which in turn permits an improved short and medium range correlation, compensated by exchange otherwise.

In principle, one could claim that the M06-L results solve the puzzle. However, the difference between adsorption sites is quite subtle, being differences of the order of a few dozens of meV. ${ }^{28}$ This order of magnitude is well within the limit accuracy of DFT methods and, in addition, other subtle effects such dispersion contribution to the adsorption energy should not be disregarded. Indeed, M06-L is known to lack a correct description of London dispersion. ${ }^{29}$ As far as we know, only a single previous study considered these non-local correlation effects on the relative stability of $\mathrm{CO}$ on top and $f c c$ hollow sites. ${ }^{30}$ These authors 
used the vdW-DFT functional of Dion et al. ${ }^{31}$ and compared the corresponding results to those obtained with the $\mathrm{PBE}^{32}$ and $\mathrm{RevPBE}^{33}$ GGA-type functionals finding that dispersion contributes to stabilize the top site relative to the $f c c$ hollow one. However, one needs to point out that a large number of van der Waals (vdW) corrections to DFT xc functionals have been proposed in the past few years and their performance on other subtle systems is far from being uniform. This is the case of graphene on Ni(111) highlighting both some significant discrepancies between results obtained from different dispersion corrected functionals and the importance of a correct treatment of this contribution when dealing with small energy differences right in the order of the hundredths of an $\mathrm{eV} .{ }^{34,35}$

From the previous discussion it is clear that even if the $\mathrm{CO} / \mathrm{Pt}(111)$ puzzle was claimed to be solved, this was well before the blooming of methods aimed at introducing dispersion terms in the xc functionals. The results of Lazic et al. ${ }^{30}$ using the DFT-vdW functional clearly show the importance of dispersion in discriminating the preference for the two types of sites. Because of this here we fully address the effect of dispersion by considering a variety of dispersion-corrected GGA and meta-GGA xc functionals well suited for the description of bulk and surface properties of transition metal ${ }^{36,37}$ revealing that vdW forces do actually contribute to bridge the gap between $f c c$ hollow and top sites of CO adsorption on $\operatorname{Pt}(111)$, yet the final effect still seems to rise from the energy level separation of CO frontier orbitals.

\section{Computational Details}

The DFT calculations have been carried out using the VASP code exploiting periodic boundary conditions. ${ }^{38}$ The projector augmented wave method was used to treat interactions between core and valence electrons. ${ }^{39}$ The PBE, ${ }^{32}$ PBEsol, ${ }^{40} \mathrm{RPBE}^{41} \mathrm{RevPBE}^{33}$ and SOGGA $11^{42}$ GGA-type functionals were considered with different types of vdW corrections. Furthermore, the non-separable gradient approximation (NGA) N12 $\mathrm{xc}_{\text {functional }}{ }^{43}$ and the TPSS $^{44}$ and M06-L ${ }^{45}$ meta-GGA functionals have also been considered, again combined with different of the available methods aimed at introducing dispersion. ${ }^{34-35}$ Among all these methods we chose Grimme D2 ${ }^{46}$ and $\mathrm{D} 3^{47}$ empirical corrections as well as the Becke-Jonson (BJ) damping on D3. ${ }^{48}$ Indeed, PBE and RPBE in conjunction with D3 or D3BJ correction is a focus to our attention as has been successfully used computational levels for the treatment of complex molecular adlayers on inorganic surfaces. ${ }^{49-52}$ Note by passing by that only twobody terms were considered, known to play a main role in finite systems, although three-body 
terms become important for some thermochemical properties already in finite systems. ${ }^{47}$ In addition, we also consider the more physically grounded many body dispersion (MBD) method of Tkatchenko and coworkers. ${ }^{53,54}$ These choices are supported from recent work showing that they provide the best treatment in systems where dispersive forces do play a key role such as graphene on $\mathrm{Ni}(111), \mathrm{H}_{2}$ storage on oxide based clusters, and many others. ${ }^{34,35,55}$ Combining the different $\mathrm{xc}$ functionals and the above commented dispersion methods results in a total of 29 methods used to explore $\mathrm{CO}$ on $\mathrm{Pt}(111)$.

In the case of $\mathrm{D} 2$ correction, we used the suggested $\mathrm{C}_{6}$ coeffcients and $\mathrm{R}_{0}$ radii as originally stated for $\mathrm{C}$ and $\mathrm{O}$ atoms, which are 1.75 and $0.7 \mathrm{~J} \mathrm{~nm}^{6} \mathrm{~mol}^{-1}$ for $\mathrm{C}_{6}$, respectively, ${ }^{46}$ whereas $\mathrm{R}_{0}$ values are 1.452 and $1.342 \AA$, respectively. For $\mathrm{Pt}$, we used $\mathrm{C}_{6}$ and $\mathrm{R}_{0}$ values of $40.62 \mathrm{~J} \mathrm{~nm}^{6} \mathrm{~mol}^{-1}$ and $1.772 \AA$, as suggested later. ${ }^{56}$ However, whereas for PBE and other xc functionals a scaling coefficient $s_{0}$ of 0.75 was used, it was set to unity in the case of TPSS functional. ${ }^{46}$ In the case of D3 correction, the functional dependent parameters $s_{6}, s_{\mathrm{r}, 6}$, and $s_{8}$ were taken from a previous benchmark, ${ }^{57}$ except for N12, which were adjusted posteriorly. ${ }^{58}$ In the case of BJ damping, $s_{6}$ parameter was set to unity, whereas $a_{1}, a_{2}$, and $s_{8}$ parameters were taken from benchmarked literature. ${ }^{48}$ As far as the MBD method ${ }^{59}$ is considered, surf values were used for densely packed $\mathrm{Pt}(111)$, in particular, $\mathrm{C}_{6}, s_{0}$, and $\mathrm{R}_{0}$ were $120,14.5$, and 2.80 , respectively, all in a.u. We refer to original studies for a definition of these variables.

The valence electrons density was expanded in a plane-wave basis set with a $415 \mathrm{eV}$ cut-off for the kinetic energy, yet test calculations for the RPBE functional using a higher cutoff of $600 \mathrm{eV}$ yield the same essentially converged results. Calculations were carried non spin-polarized for the $\operatorname{Pt}(111)$ slab, whose reciprocal space was sampled with $6 \times 6 \times 1 \Gamma$ centered k-point grid on a 54 metal atoms $(3 \times 3)$ supercell slab modeling the $(111)$ surface, comprising six atomic layers, nine atoms per layer. The three bottom layers of the slab were kept fixed during the optimizations, but at the geometry belonging to the previously optimized Pt bulk using the same method. However, the three upper layers were allowed to further relax during geometry optimizations, either for the bare surface, or in the course of adsorbed CO optimizations. A minimum vacuum region of $10 \AA$ has been placed among periodically repeated slabs in order to avoid any artificial interaction among them. Selected tests with a $20 \AA$ vacuum width affects the $E_{a d s}$ values by less than $0.01 \mathrm{eV}$. Note that during slab geometry relaxations cell parameters were kept fixed. Optimizations were performed using a Gaussian smearing of $0.2 \mathrm{eV}$ energy width to speed up convergence, yet final energy 
where extrapolated to $0 \mathrm{~K}$ (no smearing). Geometry optimizations were performed until all forces acting on relaxed atoms became less than $0.03 \mathrm{eV} \AA^{-1}$.

Focusing on top versus hollow competition for $\mathrm{CO}$ adsorption, the top site with $\mathrm{CO}$ coordinated to a surface $\mathrm{Pt}$ atom through its $\mathrm{C}$ atom has been considered whereas the facecentered cubic $(f c c)$ hollow site — with no Pt atom in the second atomic layer directly beneath the CO molecule - and the hexagonal close-packed ( $h c p$ ) hollow site — with a Pt atom in the first subsurface layer directly beneath the surface CO adsorbate; see Figure 1. Given the employed supercell, the $\mathrm{CO}$ surface coverage is of 1/9 monolayer (ML). We define the calculated $\mathrm{CO}$ adsorption energies as;

$$
E_{\mathrm{ads}}=-E_{\mathrm{CO} / \mathrm{Pt}(111)}+\left(E_{\mathrm{CO}}+E_{\mathrm{Pt}(111)}\right)
$$

where $E_{\mathrm{CO} / \mathrm{Pt}(111)}$ is the total energy of the $\mathrm{Pt}(111)$ slab model with the adsorbed $\mathrm{CO}$ molecule, $E_{\mathrm{CO}}$ is the energy of an isolated $\mathrm{CO}$ molecule, and $E_{\mathrm{Pt}(111)}$ is the energy of the optimized clean Pt (111) surface slab model. With this definition, stable adsorption/absorption corresponds to positive $E_{\text {ads }}$ values. The isolated $\mathrm{CO}$ molecule has been calculated placing it in an asymmetric box of $9 \times 10 \times 11 \AA$ and carrying the calculation at the $\Gamma$ point. Note that the adsorption energy as defined in Equation (1) is directly comparable to the heat of adsorption measured in microcalorimetry experiments ${ }^{60}$ although a proper comparison should include the difference in zero point energy (ZPE) of the gas phase and adsorbed molecule. The vibrational frequency of $\mathrm{CO}$ at top and fcc site is roughly of 2100 and $1800 \mathrm{~cm}^{-1}$, respectively. ${ }^{9}$ Hence, the effect of the ZPE in the difference of adsorption energy of CO on the $\operatorname{Pt}(111)$ surface is of less than $0.02 \mathrm{eV}$ and favoring the $f c c$ site.

\section{Results and Discussion}

The complete set of results for adsorption energy at the top and hollow sites plus the corresponding relevant structural parameters is summarized in Table 1 for the total of 29 methods explored. Additionally, calculated total energy values for the optimized structures of adsorbed $\mathrm{CO}$ and of the isolated gas phase molecule and relaxed surface model are provided in the supporting information (SI) file for all methods used in the present work. The SI also collects computational details and optimized structures, again for each one of the density functionals explored. First, we focus on the magnitude of the calculated adsorption energy values. In order to facilitate the analysis, Figure 2 shows the $E_{\text {ads }}$ values for $\mathrm{CO}$ adsorbed on top and $f c c$ hollow sites, the figure also highlights the range of experimental values spanning the $1.4-1.5 \mathrm{eV}$ interval. $7^{, 8}{ }^{, 61}$ From Figure 2 it is clear that solely RevPBE and RPBE, 
especially designed to improve the adsorption energy of $\mathrm{CO}$ on metals, ${ }^{62}$ but at the expenses of worsening the description of bulk transition metals, ${ }^{34}$ meet the experimentally measured adsorption energy. Interestingly, results from TPSS and M06-L meta-GGA functionals closely follow the RPBE prediction. However, the agreement to experiment is less satisfactory, even for RevPBE and RPBE, when considering explicitly the contribution of dispersion to the interaction. Figure 2 clearly shows that the addition of any of the here explored vdW corrections results in clear overestimation of the experimental adsorption energy by $0.3-0.5 \mathrm{eV}$.

Apart from the total value of the adsorption energy, Figure 2 also allows one to compare the calculated values of adsorption energy for on top and hollow sites. For each method, the close similarity between values for top and hollow sites predicted from the different computational approach pinpoint the underlying factors that subtly favor on site over the others. For a better visualization of this trend Figure 3 shows the difference energy ( $\left.E_{\text {diff }}\right)$ of the $E_{\text {ads }}$ of hollow sites and that of top site. With this definition, negative $E_{\text {diff }}$ values denote a higher stability than for the top site and inspection of Figure 3 shows that the majority of methods fail in reproducing the experimentally observed preference for the on top site. There are, however, some aspects worth of being commented: On one hand, note how, in average terms, dispersion brings the stability of CO on hollow sites closer to on top sites. This is true for D2, D3, and MBD corrections, yet it does not hold for D3BJ, where its application worsens the description for most cases, in line with a slightly worse description of graphene/Ni(111) interactions as earlier reported. ${ }^{35}$ Taking this into account, and focusing the attention to the PBE based cases, on observes how addition of D2 improves the description by $13 \mathrm{meV}$ in average, whereas successive treatment by D3 and MBD further stepwise improves it by 20 and $8 \mathrm{meV}$, respectively. However, the D2 correction does not always improve the description since on other GGA, NGA, or meta-GGA functionals, such as SOGGA11, RevPBE, RPBE, N12, and TPSS, the opposite trend is observed. Indeed, SOGGA11 shows relatively large $E_{\text {ads }}$ values, in line with previous studies highlighting its overbinding. ${ }^{58}$ Interestingly, the in principle more accurate D3 parameterization of dispersion leads to a proper decrease of $E_{\text {diff. }}$ In the case of RevPBE and RPBE, the description compared to pure PBE is improved by 44 and $17 \mathrm{meV}$, respectively, and it goes to $25 \mathrm{meV}$ in the case of TPSS. Notice as well the great improvement when applying the D3 correction to N12 functional, approaching the accuracy of RevPBE-D3 and RPBE-D3. Hence, present results show that relative stability of hollow and top sites for the $\mathrm{CO}$ adsorption on $\operatorname{Pt}(111)$ 
surface tends to decrease when dispersion is taken into account, in accordance to previous findings. ${ }^{30}$

An issue worth to tackle separately is that of the local M06-L functional, which has already been reported as correctly describing the preference of top versus hollow site. ${ }^{27}$ The present results further confirm this prediction, although the alleged preference for top over $f c c$ hollow by $360 \mathrm{meV}$ could not be reproduced despite using same computing package and carrying out the study at a similar low coverage. The only difference is in the energy cutoff of $415 \mathrm{eV}$ in the present work, and $500 \mathrm{eV}$ in the previous ${ }^{27}$ but also the use of slightly different surface slab model, both containing 6 atomic layers but relaxed $3+3$ in the present work and symmetrically the top and bottom two layers with the two innermost fixed in Ref. 27. The disagreement however is highly reduced when comparing PBE and RevPBE results, where the earlier work in Ref. 27 reports an $f c c$ hollow preference by 100 and $90 \mathrm{meV}$, respectively, thus much closer to present values of 169 and $49 \mathrm{meV}$, respectively with the differences in the range of numerical accuracy. Regardless of this small discrepancy, M06-L is the unique explored xc capable of favoring top over $f c c$ site. Note however that addition of vdW, on the contrary, slightly favors $f c c$ hollow by 13 and $64 \mathrm{meV}$ at D2 and D3 levels. Therefore, as far as the contribution of dispersion is concerned, only Grimme D3 correction on RPBE is capable to equal preference of top to $f c c$ hollow site, but with a too large value of the adsorption energy.

The trends in interatomic distances provide additional information regarding the performance of the 20 computational methods explored. Figure 4 shows the $\mathrm{CO}$ molecule interatomic distance when adsorbed over top site, or over the $f c c$ or hcp hollow sites, together with the experimentally derived distance from low energy electron diffraction (LEED) experiments by Ogletree et al., 6 reporting a $d(\mathrm{C}-\mathrm{O})$ distance of $1.15 \pm 0.05 \AA$. One can observe that $\mathrm{CO}$ bond length does not allow differentiating among sites. However, the situation is different when considering $d(\mathrm{C}-\mathrm{Pt})$, also shown in Figure 4 compared to those of $1.85 \pm 0.10 \AA$ also obtained from LEED. Results clearly highlight how only top site meets the structural experimentally determined data, and none of the hollow sites, whatever the computational method employed, meets this requirement. Top site is definitely the preferred to $\mathrm{CO}$, however, no significant distinction among the situations described employing various DFT xc functionals and vdW corrections is found.

Notice that, because of the problems in convergence for metallic systems and the exceedingly high computational cost when a plane wave basis set is used, hybrid functionals 
were not explicitly considered in the present study. However, as above-explained, they tend to correctly level the $2 \pi^{*} \mathrm{CO}$ orbital, and, consequently, hybrid functionals tend to describe the preference of top versus hollow sites. ${ }^{24,25}$ However, the poorer description of the delocalized band structure of metals seems to be the main reason why hybrid functionals excessively overestimate $\mathrm{CO}$ adsorption energies. Assuming that the vdW contribution to $\mathrm{E}_{\text {ads }}$ computed for GGA can be added to the results from hybrid xc functionals, this overestimation will be further increased by $\sim 0.3 \mathrm{eV}$, although it is likely that vdW effects would help in the preference of adsorption on top versus hollow by $\sim 0.05 \mathrm{eV}$.

\section{Conclusions}

In this work we investigated the role of dispersion contribution in the interaction of $\mathrm{CO}$ on $\mathrm{Pt}(111)$ with the aim to discern whether these play a key role in the preference of $\mathrm{CO}$ for atop sites of the $\operatorname{Pt}(111)$ surface. To this end, up to 29 different density functionals including or not dispersions have been considered. From the complete set of results, it appears that only M06-L is able to reproduce the correct $\mathrm{CO}$ adsorption site although only when dispersion terms are not included which indicates some error compensation effects. This claim is supported by the results of M06-L when dispersion is included which noticeably overestimate the adsorption energy. In general, inclusion of dispersion assists in bridging the gap in between top and hollow sites, and its effect is in the order of the site difference in energy for $\mathrm{CO}$ on $\mathrm{Pt}(111)$, as well as on other late transition metals, and so, yet not fully determining, it is a factor to be considered in order to get a complete unbiased description of $\mathrm{CO}$ adsorption, in particular, yet to other simple molecules in general.

Nevertheless, inclusion of dispersion leads to a consistent overestimation of the adsorption energy indicating that, in spite of an overall and confirmed success of DFT based methods on describing the interactions and energetic of reactions at surfaces, ${ }^{63}$ the accuracy of existing density functionals needs to be improved. The present results also warn on the $a d$ hoc modification of functionals to reproduce adsorption energies while neglecting physically meaningful contributions as dispersion. 


\section{Acknowledgements}

This work was supported by Spanish MINECO/FEDER grant CTQ2015-64618-R and, in part, by Generalitat de Catalunya grants (2014SGR97 and XRQTC) and from the NOMAD Center of Excellence project; the latter project has received funding from the European Union Horizon 2020 research and innovation programme under grant agreement No 676580. P.J. would like to thank the Office of the Higher Education Commission, Thailand, for supporting him with a grant under the program Strategic Scholarships for Frontier Research Network for the Ph.D. Program Thai Doctoral degree and the Graduate School of Kasetsart University. F.V. thanks the Spanish MINECO for postdoctoral contracts under the Juan de la Cierva (JCI-2010-06372) and Ramón y Cajal (RYC-2012-10129) programs, and F.I. acknowledges additional support through the ICREA Academia award for excellence in research. This work was also supported in part by the Air Force Office of Scientific Research under grant number FA9550-11-1-0078.

\section{Supporting information}

The Supporting Information is available free of charge on the ACS Publications website at DOI: $\operatorname{xxxxxxxxxxxxxx}$

Table S1. Total energy of CO, relaxed slab model of $\operatorname{Pt}(111)$ and of the optimized structure of $\mathrm{CO}$ adsorbed at the surface model as predicted by the different functionals used in the present work. All values are in $\mathrm{eV}$.

Computationals details (INCAR files): Default parameters and Functional settings Structural information: Optimized geomtr for each fucntional (CONTCAR files) 
Figure 1. Top view of CO adsorption sites on Pt(111) surface, including a) top, b) hcp and c) fcc hollow sites. $\mathrm{Pt}, \mathrm{C}$, and $\mathrm{O}$ atoms positions are denoted by cyan, orange, and red spheres, respectively.

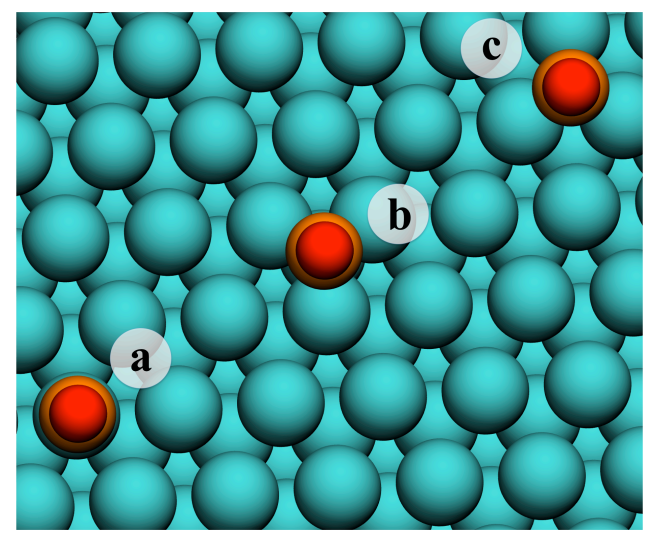


Figure 2. Adsorption ( $\left.E_{\text {ads }}\right)$ energies, for $\mathrm{CO}$ adsorbed on $f c c, h c p$, or top sites on $\operatorname{Pt}(111)$ surfaces as obtained employing various DFT xc functionals, including or not a treatment of dispersive force. Experimental range of values is delimited by a yellowish region.

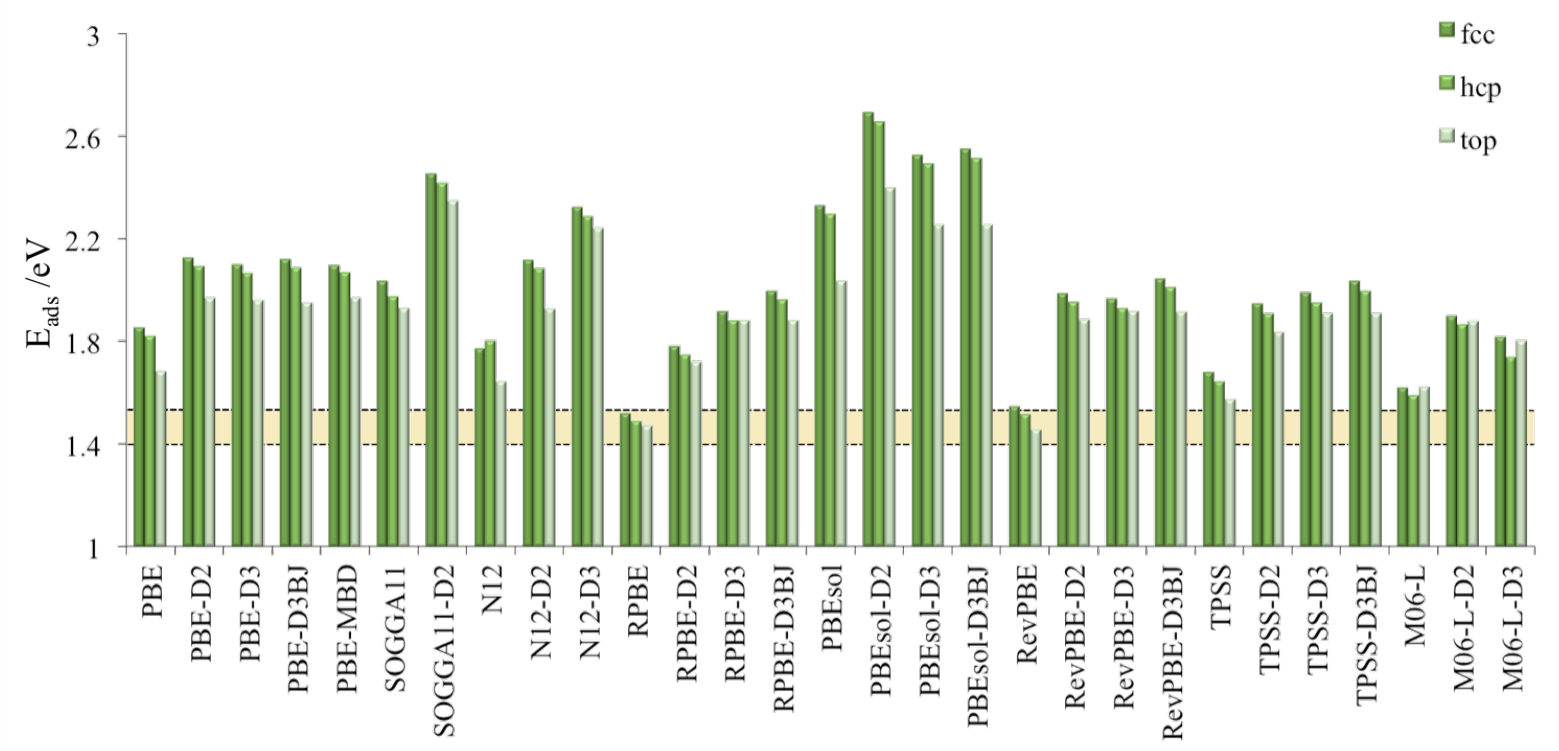


Figure 3. Difference in adsorption energies $\left(E_{\text {diff }}\right)$ in between hollow and top site for $\mathrm{CO}$ adsorption on $\operatorname{Pt}(111)$ surface, as obtained employing various DFT xc functionals, including or not a treatment of dispersive forces.

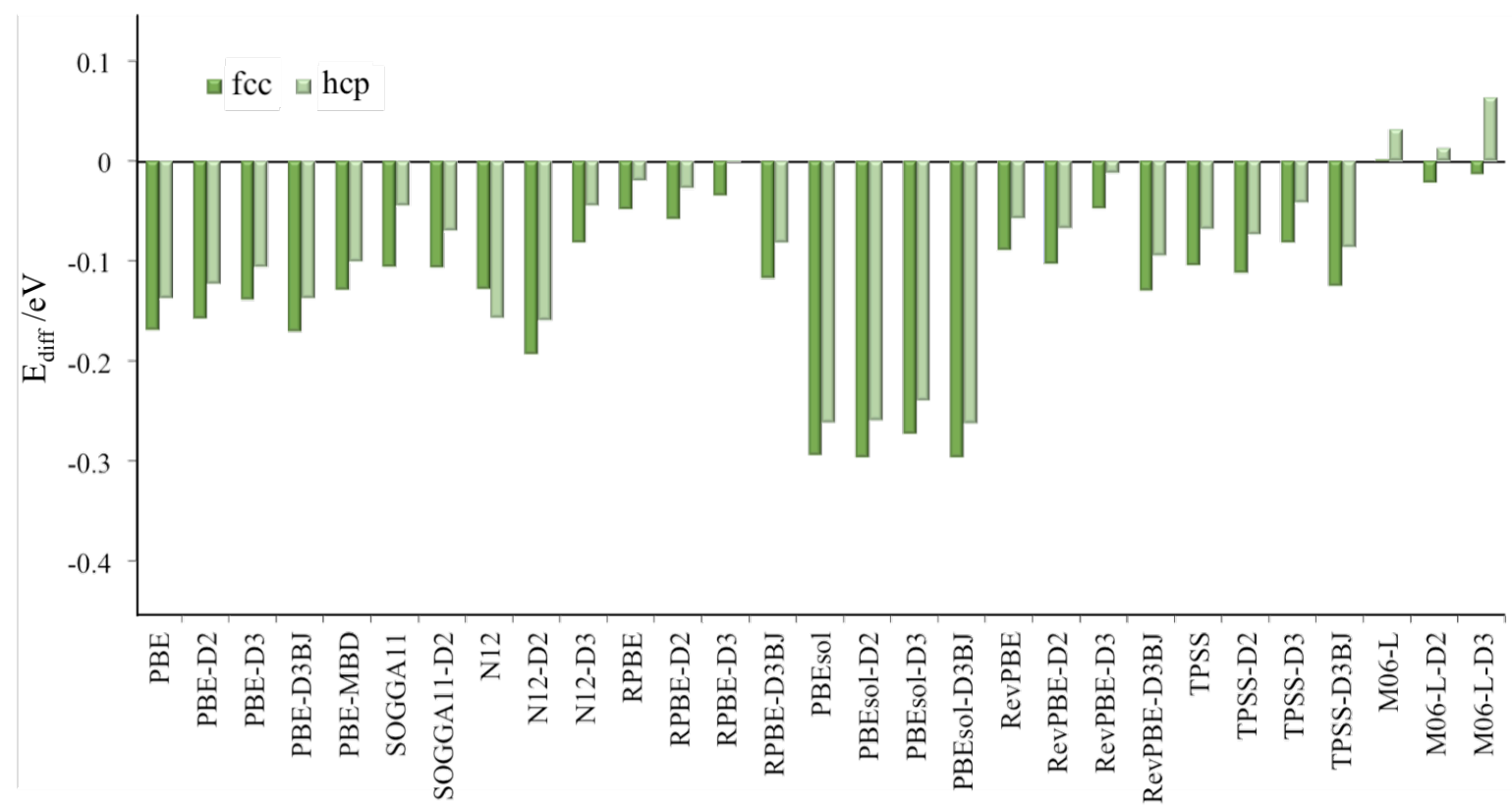


Figure 4. Interatomic $\mathrm{CO}$ molecule distance, $d(\mathrm{C}-\mathrm{O})$ (top panel), when $\mathrm{CO}$ adsorbed on top site of a Pt surface atom of $\operatorname{Pt}(111)$, or over $f c c$ or $h c p$ hollow sites, as well the distance among $\mathrm{C}$ atom and those surface $\mathrm{Pt}$ atoms involved in its adsorption, $d(\mathrm{C}-\mathrm{Pt})$ (bottom panel), as obtained employing various DFT xc functionals, including or not a treatment of dispersive forces. Experimental range of values is delimited by a yellowish region.
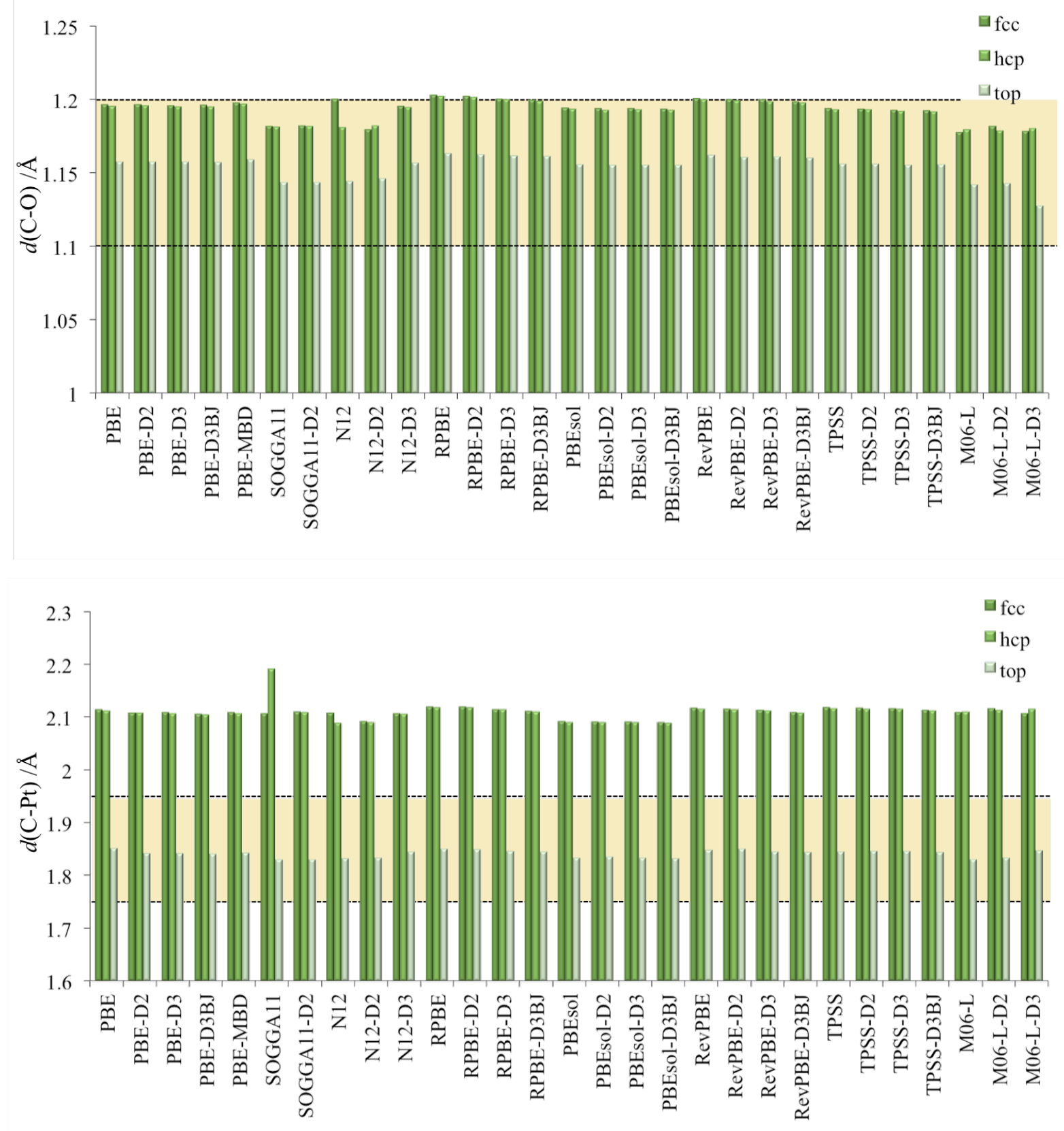
Table 1. Adsorption energy ( $E_{\text {ads }}$ in $\left.\mathrm{eV}\right)$ and relevant distances in $\AA$ for $\mathrm{CO}$ adsorbed on top, $f c c$ or $h c p$ hollow sites of the surface $\mathrm{Pt}(111)$ as predicted from difference $\mathrm{xc}$ functionals, including or not a treatment of dispersive forces.

\begin{tabular}{|c|c|c|c|c|}
\hline Method & Site & $E_{\text {ads }}$ & $d_{\mathrm{C}-\mathrm{O}}$ & $d_{\mathrm{C}-\mathrm{Pt}}$ \\
\hline \multirow[t]{3}{*}{ PBE } & $f c c$ & 1.854 & 1.196 & 2.114 \\
\hline & hcp & 1.822 & 1.195 & 2.112 \\
\hline & top & 1.685 & 1.157 & 1.851 \\
\hline \multirow[t]{3}{*}{ PBE-D2 } & $f c c$ & 2.128 & 1.197 & 2.108 \\
\hline & hcp & 2.093 & 1.196 & 2.107 \\
\hline & top & 1.970 & 1.158 & 1.842 \\
\hline \multirow[t]{3}{*}{ PBE-D3 } & $f c c$ & 2.099 & 1.196 & 2.108 \\
\hline & hcp & 2.066 & 1.195 & 2.107 \\
\hline & top & 1.960 & 1.158 & 1.841 \\
\hline \multirow[t]{3}{*}{ PBE-D3BJ } & $f c c$ & 2.121 & 1.196 & 2.106 \\
\hline & hcp & 2.088 & 1.195 & 2.105 \\
\hline & top & 1.951 & 1.157 & 1.841 \\
\hline \multirow[t]{3}{*}{ PBE-MBD } & $f c c$ & 2.097 & 1.198 & 2.109 \\
\hline & hcp & 2.068 & 1.197 & 2.107 \\
\hline & top & 1.968 & 1.159 & 1.842 \\
\hline \multirow[t]{3}{*}{ SOGGA11 } & $f c c$ & 2.036 & 1.182 & 2.107 \\
\hline & hcp & 1.974 & 1.181 & 2.192 \\
\hline & top & 1.930 & 1.143 & 1.830 \\
\hline \multirow[t]{3}{*}{ SOGGA11-D2 } & $f c c$ & 2.454 & 1.182 & 2.110 \\
\hline & hcp & 2.417 & 1.182 & 2.109 \\
\hline & top & 2.348 & 1.144 & 1.830 \\
\hline N12 & $f c c$ & 1.773 & 1.200 & 2.108 \\
\hline
\end{tabular}




\begin{tabular}{|c|c|c|c|c|}
\hline & hcp & 1.802 & 1.181 & 2.088 \\
\hline & top & 1.645 & 1.144 & 1.832 \\
\hline \multirow[t]{3}{*}{ N12-D2 } & $f c c$ & 2.119 & 1.180 & 2.092 \\
\hline & hcp & 2.085 & 1.182 & 2.089 \\
\hline & top & 1.926 & 1.146 & 1.833 \\
\hline \multirow[t]{3}{*}{ N12-D3 } & $f c c$ & 2.325 & 1.195 & 2.106 \\
\hline & hcp & 2.288 & 1.195 & 2.106 \\
\hline & top & 2.243 & 1.157 & 1.845 \\
\hline \multirow[t]{3}{*}{ RPBE } & $f c c$ & 1.521 & 1.203 & 2.120 \\
\hline & hcp & 1.492 & 1.202 & 2.118 \\
\hline & top & 1.472 & 1.163 & 1.850 \\
\hline \multirow[t]{3}{*}{ RPBE-D2 } & $f c c$ & 1.781 & 1.202 & 2.120 \\
\hline & hcp & 1.749 & 1.202 & 2.118 \\
\hline & top & 1.722 & 1.162 & 1.850 \\
\hline \multirow[t]{3}{*}{ RPBE-D3 } & $f c c$ & 1.918 & 1.200 & 2.114 \\
\hline & hcp & 1.883 & 1.200 & 2.114 \\
\hline & top & 1.883 & 1.162 & 1.846 \\
\hline \multirow[t]{3}{*}{ RPBE-D3BJ } & $f c c$ & 1.997 & 1.200 & 2.111 \\
\hline & hcp & 1.962 & 1.199 & 2.110 \\
\hline & top & 1.880 & 1.161 & 1.845 \\
\hline \multirow[t]{3}{*}{ PBEsol } & $f c c$ & 2.330 & 1.194 & 2.091 \\
\hline & hcp & 2.297 & 1.193 & 2.090 \\
\hline & top & 2.036 & 1.156 & 1.833 \\
\hline PBEsol-D2 & $f c c$ & 2.694 & 1.194 & 2.090 \\
\hline
\end{tabular}




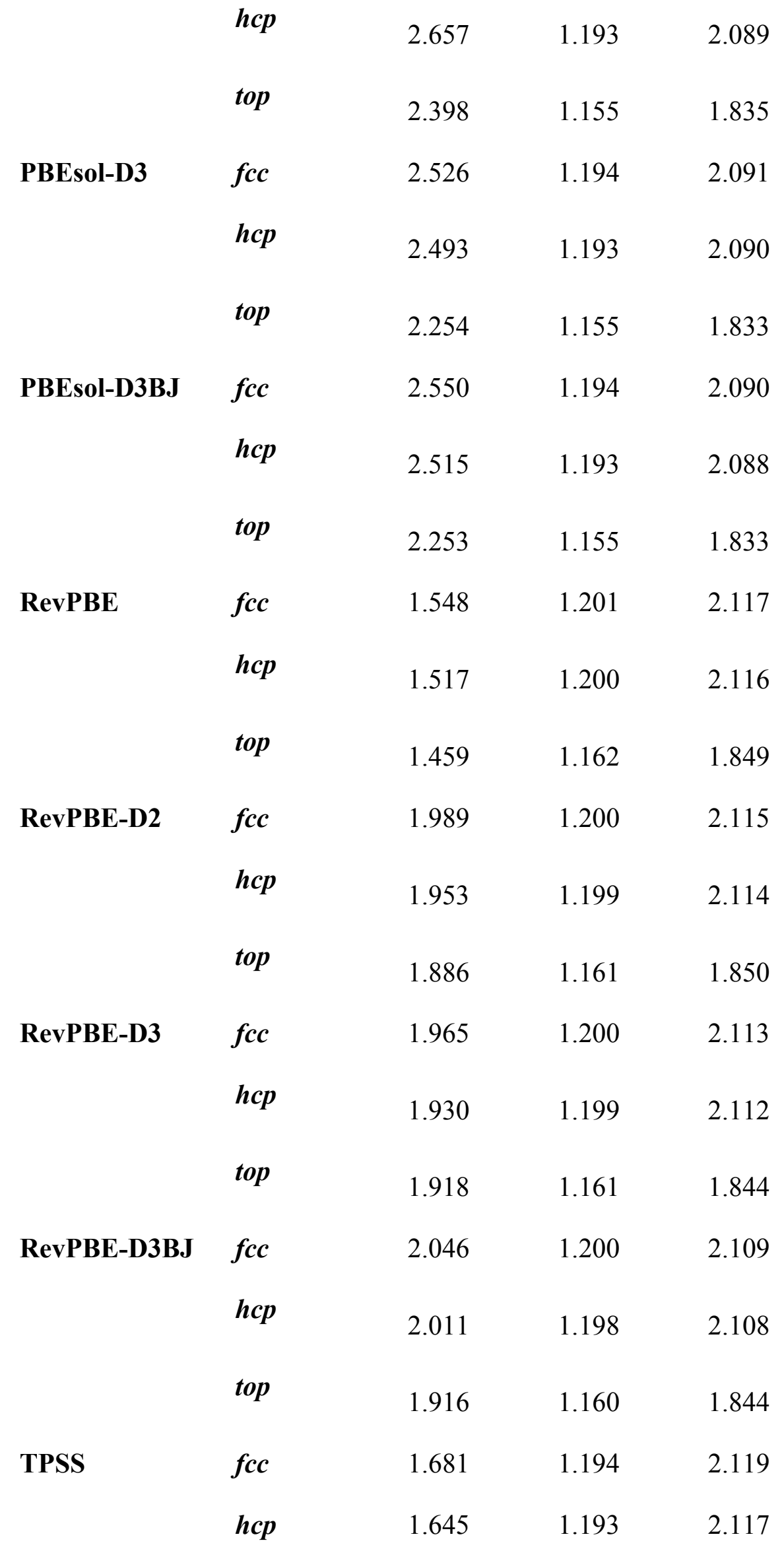




\begin{tabular}{|c|c|c|c|c|}
\hline & top & 1.576 & 1.156 & 1.844 \\
\hline \multirow[t]{3}{*}{ TPSS-D2 } & $f c c$ & 1.949 & 1.193 & 2.117 \\
\hline & hcp & 1.910 & 1.193 & 2.115 \\
\hline & top & 1.837 & 1.156 & 1.846 \\
\hline \multirow[t]{3}{*}{ TPSS-D3 } & $f c c$ & 1.990 & 1.193 & 2.117 \\
\hline & hcp & 1.951 & 1.192 & 2.115 \\
\hline & top & 1.909 & 1.155 & 1.846 \\
\hline \multirow[t]{3}{*}{ TPSS-D3BJ } & $f c c$ & 2.035 & 1.192 & 2.113 \\
\hline & hcp & 1.996 & 1.192 & 2.112 \\
\hline & top & 1.910 & 1.156 & 1.844 \\
\hline \multirow[t]{3}{*}{ M06-L } & $f c c$ & 1.622 & 1.178 & 2.108 \\
\hline & hcp & 1.592 & 1.180 & 2.110 \\
\hline & top & 1.624 & 1.142 & 1.830 \\
\hline \multirow[t]{3}{*}{ M06-L-D2 } & $f c c$ & 1.900 & 1.182 & 2.116 \\
\hline & hcp & 1.865 & 1.179 & 2.114 \\
\hline & top & 1.878 & 1.143 & 1.833 \\
\hline \multirow[t]{3}{*}{ M06-L-D3 } & $f c c$ & 1.818 & 1.178 & 2.107 \\
\hline & hcp & 1.740 & 1.180 & 2.115 \\
\hline & top & 1.804 & 1.127 & 1.847 \\
\hline
\end{tabular}




\section{References}

${ }^{1}$ Frenking, G. Theoretical Aspects of Transition Metal Catalysis. Springer Berlin Heidelberg: 2005.

2 Thomas, J. M.; Thomas, W. J. Principles and Practice of Heterogeneous Catalysis. 2 ed.; Wiley-VCH: Weinheim, 2015.

${ }^{3}$ Anderson, J. A.; Fernández-García, M. Supported Metals in Catalysis Imperial College Press: London, 2011.

${ }^{4} \mathrm{Wu}$, J.; Yang, H. Platinum-Based Oxygen Reduction Electrocatalysts. Acc. Chem. Res. 2013, 46, 1848-1857.

${ }^{5}$ Hopster, H.; Ibach, H. Adsorption of CO on Pt(111) and Pt 6(111) $\times(111)$ Studied by High Resolution Electron Energy Loss Spectroscopy and Thermal Desorption Spectroscopy. Surf. Sci. 1978, 77, 109-117.

${ }^{6}$ Ogletree, D. F.; Van Hove, M. A.; Somorjai, G. A. LEED Intensity Analysis of the Structures of Clean $\mathrm{Pt}(111)$ and of CO Adsorbed on $\mathrm{Pt}(111)$ in the $\mathrm{c}(4 \times 2)$ Arrangement. Surf. Sci. 1986, 173, 351-365.

${ }^{7}$ Ertl, G.; Neumann, M.; Streit, K. M. Chemisorption of CO on the Pt(111) surface. Surf. Sci. 1977, 64, 393-410.

${ }^{8}$ Golibrzuch, K.; Shirhatti, P. R.; Geweke, J.; Werdecker, J.; Kandratsenka, A.; Auerbach, D. J.; Wodtke, A. M.; Bartels, C. CO Desorption from a Catalytic Surface: Elucidation of the Role of Steps by Velocity-Selected Residence Time Measurements. J. Am. Chem. Soc 2015, 137, 1465-1475.

${ }^{9}$ Steininger, H.; Lehwald, S.; Ibach, H. On the adsorption of CO on Pt(111). Surf. Sci. 1982, $123,264-282$.

${ }^{10}$ 10. Blackman, G. S.; Xu, M. L.; Ogletree, D. F.; Van Hove, M. A.; Somorjai, G. A. Mix of Molecular Adsorption Sites Detected for Disordered CO on Pt(111) by Diffuse Low-Energy Electron Diffraction. Phys. Rev. Lett. 1988, 61, 2352-2355.

11 Stroscio, J. A.; Eigler, D. M. Atomic and Molecular Manipulation with the Scanning Tunneling Microscope. Science 1991, 254, 1319-1326.

${ }^{12}$ Feibelman, P. J.; Hammer, B.; Nørskov, J. K.; Wagner, F.; Scheffler, M.; Stumpf, R.; Watwe, R.; Dumesic, J. The CO/Pt(111) Puzzle. J. Phys. Chem. B 2001, 105, 4018-4025.

${ }^{13}$ Grinberg, I.; Yourdshahyan, Y.; Rappe, A. M. CO on Pt(111) puzzle: A possible solution. J. Chem. Phys. 2002, 117, 2264-2270. 
${ }^{14}$ Olsen, R. A.; Philipsen, P. H. T.; Baerends, E. J. CO on Pt(111): A Puzzle Revisited. J. Chem. Phys. 2003, 119, 4522-4528.

${ }^{15}$ Kresse, G.; Gil, A.; Sautet, P. Significance of Single-Electron Energies for the Description of CO on Pt(111). Phys. Rev. B 2003, 68, 073401.

${ }^{16}$ Gil, A.; Clotet, A.; Ricart, J. M.; Kresse, G.; Garcı-Hernandez, M.; Rösch, N.; Sautet, P. Site Preference of CO Chemisorbed on Pt(111) from Density Functional Calculations. Surf. Sci. 2003, 530, 71-87.

${ }^{17}$ Blyholder, G. Molecular Orbital View of Chemisorbed Carbon Monoxide. J. Phys. Chem. 1964, 68, 2772-2777.

${ }^{18}$ Soini, T. M.; Genest, A.; Rösch, N. Assessment of Hybrid Density Functionals for the Adsorption of Carbon Monoxide on Platinum Model Clusters. J. Phys. Chem. A 2015, 119, 4051-4056.

${ }^{19}$ Stroppa, A.; Termentzidis, K.; Paier, J.; Kresse, G.; Hafner, J. CO Adsorption on Metal Surfaces: A Hybrid Functional Study with Plane-Wave Basis Set. Phys. Rev. B 2007, 76, 195440.

${ }^{20}$ Schimka, L.; Harl, J.; Stroppa, A.; Grüneis, A.; Marsman, M.; Mittendorfer, F.; Kresse, G. Accurate Surface and Adsorption Energies from Many-Body Perturbation Theory. Nat Mater 2010, 9, 741-744.

${ }^{21}$ Adamo, C.; Barone, V. Toward Reliable Density Functional Methods without Adjustable Parameters: The PBE0 Model. J. Chem. Phys. 1999, 110, 6158-6170.

${ }^{22} 22$. Becke, A. D. Density functional Thermochemistry. III. The Role of Exact Exchange. J. Chem. Phys. 1993, 98, 5648-5652.

${ }^{23}$ Paier, J.; Marsman, M.; Hummer, K.; Kresse, G.; Gerber, I. C.; Ángyán, J. G. Screened Hybrid Density Functionals Applied to Solids. J. Chem. Phys. 2006, 124, 154709.

${ }^{24}$ Wang, Y.; de Gironcoli, S.; Hush, N. S.; Reimers, J. R. Successful a Priori Modeling of CO Adsorption on Pt(111) Using Periodic Hybrid Density Functional Theory. J Am. Chem. Soc. 2007, 129, 10402-10407.

${ }^{25}$ Stroppa, A.; Kresse, G. The Shortcomings of Semi-Local and Hybrid Functionals: What We Can Learn from Surface Science Studies. New J. Phys. 2008, 10, 063020.

${ }^{26}$ Orita, H.; Itoh, N.; Inada, Y. All Electron Scalar Relativistic Calculations on Adsorption of $\mathrm{CO}$ on $\mathrm{Pt}(111)$ with Full-Geometry Optimization: a Correct Estimation for CO SitePreference. Chem. Phys. Lett. 2004, 384, 271-276. 
${ }^{27}$ Luo, S.; Zhao, Y.; Truhlar, D. G. Improved CO Adsorption Energies, Site Preferences, and Surface Formation Energies from a Meta-Generalized Gradient Approximation ExchangeCorrelation Functional, M06-L. J. Phys. Chem. Lett. 2012, 3, 2975-2979.

${ }^{28}$ Schweizer, E.; Persson, B. N. J.; Tüshaus, M.; Hoge, D.; Bradshaw, A. M. The Potential Energy Surface, Vibrational Phase Relaxation and the Order-Disorder Transition in the Adsorption System Pt\{111\}-CO. Surf. Sci. 1989, 213, 49-89.

${ }^{29}$ Goerigk, L.; Kruse, H.; Grimme, S. Benchmarking Density Functional Methods against the S66 and S66x8 Datasets for Non-Covalent Interactions. ChemPhysChem 2011, 12, 34213433 .

${ }^{30}$ Lazić, P.; Alaei, M.; Atodiresei, N.; Caciuc, V.; Brako, R.; Blügel, S. Density Functional Theory with Nonlocal Correlation: A Key to the Solution of the CO Adsorption Puzzle. Phys. Rev. B 2010, $81,045401$.

${ }^{31}$ Dion, M.; Rydberg, H.; Schröder, E.; Langreth, D. C.; Lundqvist, B. I. Van der Waals Density Functional for General Geometries. Phys. Rev. Lett. 2004, 92, 246401.

${ }^{32}$ Perdew, J. P.; Burke, K.; Ernzerhof, M. Generalized Gradient Approximation Made Simple. Phys. Rev. Lett. 1996, 77, 3865-3868.

${ }^{33}$ Zhang, Y.; Yang, W. Comment on "Generalized Gradient Approximation Made Simple”'. Phys. Rev. Lett. 1998, 80, 890-890.

${ }^{34}$ Janthon, P.; Viñes, F.; Kozlov, S. M.; Limtrakul, J.; Illas, F. Theoretical Assessment of Graphene-Metal Contacts. J. Chem. Phys. 2013, 138, 244701.

${ }^{35}$ Muñoz-Galán, H.; Viñes, F.; Gebhardt, J.; Görling, A.; Illas, F. The Contact of Graphene with Ni(111) Surface: Description by Modern Dispersive Forces Approaches. Theoret. Chem. Acc. 2016, 135, 165.

${ }^{36}$ Janthon, P.; Kozlov, S. M.; Viñes, F.; Limtrakul, J.; Illas, F. Establishing the Accuracy of Broadly Used Density Functionals in Describing Bulk Properties of Transition Metals. $J$. Chem. Theory Comput. 2013, 9, 1631-1640.

${ }^{37}$ Janthon, P.; Luo, S.; Kozlov, S. M.; Viñes, F.; Limtrakul, J.; Truhlar, D. G.; Illas, F. Bulk Properties of Transition Metals: A Challenge for the Design of Universal Density Functionals. J. Chem. Theory Comput. 2014, 10, 3832-3839.

${ }^{38}$ Kresse, G.; Furthmüller, J. Efficiency of Ab-Initio Total Energy Calculations for Metals and Semiconductors Using a Plane-Wave Basis Set. Comput. Mater. Sci. 1996, 6, 15-50. ${ }^{39}$ Blöchl, P. E. Projector Augmented-Wave Method. Phys. Rev. B 1994, 50, 17953-17979. 
${ }^{40}$ Perdew, J. P.; Ruzsinszky, A.; Csonka, G. I.; Vydrov, O. A.; Scuseria, G. E.; Constantin, L. A.; Zhou, X.; Burke, K. Restoring the Density-Gradient Expansion for Exchange in Solids and Surfaces. Phys. Rev. Lett. 2008, 100, 136406.

${ }^{41}$ Hammer B.; Hansen, L. B.; Nørskov, J. K. Improved Adsorption Energetics within Density-Functional Theory Using Revised Perdew-Burke-Ernzerhof Functionals. Phys. Rev. $B$ 1999, 59, 7413

${ }^{42}$ Peverati, R.; Zhao, Y.; Truhlar, D. G. Generalized Gradient Approximation That Recovers the Second-Order Density-Gradient Expansion with Optimized Across-the-Board Performance. J.Phys.Chem. Lett. 2011, 2, 1991-1997.

${ }^{43}$ Peverati, R.; Truhlar, D. G. Exchange-Correlation Functional with Good Accuracy for Both Structural and Energetic Properties while Depending Only on the Density and Its Gradient. J. Chem. Theory Comput. 2012, 8, 2310-2319.

${ }^{44}$ Tao, J.; Perdew, J. P.; Staroverov, V. N.; Scuseria, G. E. Climbing the Density Functional Ladder: Nonempirical Meta-Generalized Gradient Approximation Designed for Molecules and Solids. Phys. Rev. Lett. 2003, 91, 146401.

45 Zhao, Y.; Truhlar, D. G. A New Local Density Functional for Main-Group Thermochemistry, Transition Metal Bonding, Thermochemical Kinetics, and Noncovalent Interactions. J. Chem. Phys. 2006, 125, 194101.

${ }^{46}$ Grimme, S. Semiempirical GGA-Type Density Functional Constructed with a Long-Range Dispersion Correction. J. Comput. Chem. 2006, 27, 1787-1799.

47 Grimme, S.; Antony, J.; Ehrlich, S.; Krieg, H. A Consistent and Accurate Ab Initio Parametrization of Density Functional Dispersion Correction (DFT-D) for the 94 Elements H-Pu. J. Chem. Phys. 2010, 132, 154104.

${ }^{48}$ Grimme, S.; Ehrlich, S.; Goerigk, L. Effect of the Damping Function in Dispersion Corrected Density Functional Theory. J. Comput. Chem. 2011, 32, 1456-1465.

${ }^{49}$ Reimers, J. R.; Panduwinata, D.; Visser, J.; Chin, Y.; Tang, C.; Goerigk, L.; Ford, M. J.; Sintic, M.; Sum, T.-J.; Coenen, M. J. J.; et al. A Priori Calculations of the Free Energy of Formation from Solution of Polymorphic Self-Assembled Monolayers. Proc. Natl. Acad. Sci. 2015, 112, E6101-E6110.

${ }^{50}$ Roman, T.; Groß, A. Polymorphism of Water in Two Dimensions. J. Phys. Chem. C 2016, $120,13649-13655$.

${ }^{51}$ Reimers, J. R.; Panduwinata, D.; Visser, J.; Chin, Y.; Tang, C.; Goerigk, L.; Ford, M. J.; Baker, M.; Sum, T. J.; Coenen, M. J. J.; et al. From Chaos to Order: Chain-Length 
Dependence of the Free Energy of Formation of Meso-Tetraalkylporphyrin Self-Assembled Monolayer Polymorphs. J. Phys. Chem. C 2016, 120, 1739-1748.

${ }^{52}$ Weber, H.; Bredow, T.; Kirchner, B. Adsorption Behavior of the 1,3-Dimethylimidazolium Thiocyanate and Tetracyanoborate Ionic Liquids at Anatase (101) Surface. J. Phys. Chem. C 2015, 119, 15137-15149.

${ }^{53}$ Tkatchenko, A.; DiStasio, R. A.; Car, R.; Scheffler, M. Accurate and Efficient Method for Many-Body van der Waals Interactions. Phys. Rev. Lett. 2012, 108, 236402.

${ }^{54}$ Ambrosetti, A.; Reilly, A. M.; DiStasio, R. A.; Tkatchenko, A. Long-Range Correlation Energy Calculated from Coupled Atomic Response Functions. J. Chem. Phys. 2014, 140, 18A508.

${ }^{55}$ Gebhardt, J.; Vines, F.; Bleiziffer, P.; Hieringer, W.; Gorling, A. Hydrogen Storage on Metal Oxide Model Clusters using Density-Functional Methods and Reliable van der Waals Corrections. Phys. Chem. Chem. Phys. 2014, 16, 5382-5392.

${ }^{56}$ Martin, A.; Sébastien, L.; Olle, E.; Natalia, V. S. Adsorption of $\mathrm{Cu}$, Ag, and Au Atoms on Graphene Including van der Waals Interactions. J. Phys.: Cond. Matt 2011, 23, 395001.

57 Goerigk, L.; Grimme, S. A thorough Benchmark of Density Functional Methods for General Main Group Thermochemistry, Kinetics, and Noncovalent Interactions. Phys. Chem. Chem. Phys. 2011, 13, 6670-6688.

58 Goerigk, L. Treating London-Dispersion Effects with the Latest Minnesota Density Functionals: Problems and Possible Solutions. J. Phys. Chem. Lett. 2015, 6, 3891-3896.

${ }^{59}$ Ambrosetti, A.; Reilly, A. M.; Di Stasio Jr., R. A.; Tkatchenko, A. Long-Range Correlation Energy Calculated from Coupled Atomic Response Functions J. Chem. Phys. 2014, 140, 18A508

${ }^{60}$ Crowe, M. C.; Campbell, C. T. Adsorption Microcalorimetry: Recent Advances in Instrumentation and Application. Annu. Rev. Anal. Chem. 2011, 4, 41-58.

${ }^{61}$ Campbell, C. T.; Ertl, G.; Kuipers, H.; Segner, J. A Molecular Beam Investigation of the Interactions of CO with a Pt(111) Surface. Surf. Sci. 1981, 107, 207-219.

${ }^{62}$ Hammer, B.; Hansen, L. B.; Nørskov, J. K. Improved Adsorption Energetics within Density-Functional Theory using Revised Perdew-Burke-Ernzerhof Functionals. Phys. Rev. B 1999, 59, 7413-7421.

${ }^{63}$ Wellendorff, J.; Silbaugh, T. L.; Garcia-Pintos, D.; Nørskov, J. K.; Bligaard, T.; Studt, F.; Campbell, C. T. A Benchmark Database for Adsorption Bond Energies to Transition Metal Surfaces and Comparison to Selected DFT Functionals. Surf. Sci, 2015, 640, 36-44. 
Graphic for TOC

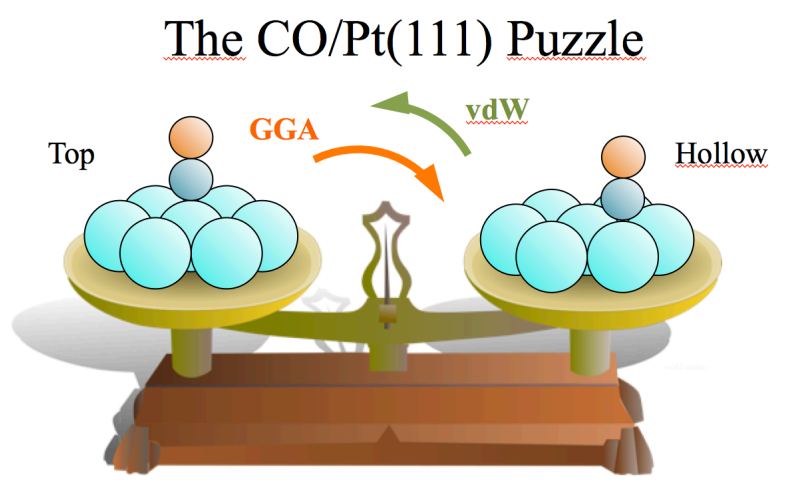

